\title{
Synergistic effect of scaffold composition and dynamic culturing environment in multilayered systems for bone tissue engineering
}

\author{
Márcia T. Rodrigues ${ }^{1,2}$, Albino Martins ${ }^{1,2}$, Isabel R. Dias ${ }^{1,2,3}$, Carlos A. Viegas ${ }^{1,2,3}$, Nuno M. Neves ${ }^{1,2}$, \\ Manuela E. Gomes ${ }^{1,2}$ and Rui L. Reis ${ }^{1,2 *}$ \\ ${ }^{1} 3$ B's Research Group - Biomaterials, Biodegradables and Biomimetics, University of Minho, Headquarters of the European Institute of \\ Excellence on Tissue Engineering and Regenerative Medicine, AvePark, 4806-909 Taipas, Guimarães, Portugal \\ ${ }^{2}$ ICVS/3B's - PT Government Associate Laboratory, Braga/Guimarães, Portugal \\ ${ }^{3}$ Department of Veterinary Sciences, University of Trás-os-Montes e Alto Douro, Quinta dos Prados, 5000-911 Vila Real, Portugal
}

\begin{abstract}
Bone extracellular matrix (ECM) is composed of mineralized collagen fibrils which support biological apatite nucleation that participates in bone outstanding properties. Understanding and mimicking bone morphological and physiological parameters at a biological scale is a major challenge in tissue engineering scaffolding. Using emergent (nano)technologies scaffold designing may be critically improved, enabling highly functional tissue substitutes for bone applications. This study aims to develop novel biodegradable composite scaffolds of tricalcium phosphate (TCPs) and electrospun nanofibers of poly( $\epsilon$-caprolactone) (PCL), combining TCPs osteoconductivity with PCL biocompatibility and elasticity, mimicking bone structure and composition. We hypothesized that scaffolds with such structure/composition would stimulate the proliferation and differentiation of bone marrow stromal cells (BMSCs) towards the osteogenic phenotype. Composite scaffolds, developed by electrospining using consecutive stacked layers of PCL and TCPs, were characterized by FTIR spectroscopy, X-Ray diffraction and scanning electronic microscopy. Cellular behavior was assessed in goat BMSCs seeded onto composite scaffolds and cultured in static or dynamic conditions, using basal or osteogenic media during 7, 14 or 21 days. Cellular proliferation was quantified and osteogenic differentiation confirmed by alkaline phosphatase activity, alizarin red staining and immunocytochemistry for osteocalcin and collagen I. Results suggest that PCL-TCP scaffolds provide a 3D support for gBMSCs proliferation and osteogenic differentiation with production of ECM. TCPs positively stimulate the osteogenic process, especially under dynamic conditions, where PCL-TCP scaffolds are sufficient to promote osteogenic differentiation even in basal medium conditions. The enhancement of the osteogenic potential in dynamic conditions evidences the synergistic effect of scaffold composition and dynamic stimulation in gBMSCs osteogenic differentiation. Copyright (c) 2012 John Wiley \& Sons, Ltd.
\end{abstract}

Received 13 May 2011; Accepted 13 July 2011

\section{Introduction}

Bone fracture defects, bone loss, infection or bone tumoral resections are serious problems that require bone surgery. The implantation of auto- or allografts has long been used

*Correspondence to: Rui L. Reis, 3B's Research Group Biomaterials, Biodegradables and Biomimetics, University of Minho, Headquarters of the European Institute of Excellence on Tissue Engineering and Regenerative Medicine, AvePark, 4806-909 Taipas, Guimarães, Portugal.E-mail: rgreis@gmail.com as a standard procedure for bone reconstruction. Nevertheless, graft strategies are associated with significant disadvantages, namely tissue morbidity at the harvesting site, donor scarcity, and immune response, and disease transmissions. To overcome these limitations, the challenge remains to design novel tissue engineered products. The selection of biomaterials plays a key role in the development of an engineered product aimed at tissue regeneration and repair, since materials can tailor the biophysical and biochemical milieu at a molecular level, directing cellular behaviour and function. 
Synthetic polymers such as poly e-caprolactone (PCL) are typically more versatile for tailoring a wide range of properties and structure features. In addition, they represent a reliable source of raw materials with adequate mechanical properties and are able to avoid immunogenicity problems (Tsuji and Ikada 1996; Bezwada et al., 1995). Nevertheless, polymers lack properties to stimulate biological functions such as osteoconductivity and cell bioactivity (Rezwan et al., 2006). Concerning these properties, ceramic materials offer advantages over polymers, but their high modulus and brittle behaviour (Collins et al., 2009) limit their processability into 3D scaffolds (Yang et al., 2001; Chen et al., 2002).

Designing scaffolds using nanotechnology tools aims at mimicking the extracellular matrix (ECM) structure and complexity at a biological scale, since natural bone matrix is mainly composed of an intricate nanofiber structure of nonstoichometric hydroxyapatite integrated in collagen fibers. At a submicron level, this organic-inorganic combination confers the intrinsic unique biomechanical and functional properties of bone 3D architecture.

The products of electrospining technology are microand nanostructured scaffolds made of an ultrafine and continuous fibre network with variable pore size distribution, high microporosity, and high surface-to-volume ratio, and are morphologically similar to natural ECM (Martins et al., 2008). Several materials, including synthetic and natural origin polymers (Ngiam et al., 2009; Ko et al., 2008; Srouji et al., 2008; Hosseinkhani et al., 2006; Araujo et al., 2008; Gupta et al., 2009; Li et al., 2005b; Shin et al., 2004; Xin et al., 2007) and proteins (Ngiam et al., 2009; Srouji et al., 2008; Hosseinkhani et al., 2006; Li et al., 2005a; Sefcik et al., 2008), have been successfully electrospun into nanofiber scaffolds, which interacted positively with intercellular communications by sustaining cell adhesion, proliferation, and differentiation towards the osteogenic phenotype both in vitro (Ko et al., 2008; Srouji et al., 2008; Li et al., 2005b; Xin et al., 2007; Sefcik et al., 2008) and in vivo (Ko et al., 2008; Srouji et al., 2008; Shin et al., 2004).

Considering the properties and flexibility of electrospun nanofiber meshes, the incorporation of biologically active factors such as matrix proteins (Li et al., 2006) or minerals such as calcium phosphates (Ngiam et al., 2009; Ko et al., 2008; Araujo et al., 2008; Gupta et al., 2009), either in the spun fibers or as coatings, makes them attractive for improving scaffold designs for bony functional substitutes. Moreover, an integrated composite scaffold of a polymer with a ceramic material offers a chemical environment that would resemble the organic and inorganic components of bone native matrix (Zhang et al., 2007).

This study formulated a novel multilayer composite structure combining the osteoinductive properties of beta-tricalcium phosphate powder with a degradable polymer. PCL was selected due to its degradability and cellular compatibility and because this material has been widely studied for biomedical applications (Srouji et al., 2008; Araujo et al., 2008; Gupta et al., 2009; Li et al., 2005b; Shin et al., 2004). Several studies have suggested hydroxyapatite as the ceramic material preferred for bone strategies (Ngiam et al., 2009; Li et al., 2006; Gupta et al., 2009). Nevertheless, the osteoconductive and bioabsorbable nature of beta-tricalcium phosphate together with an earlier incorporation into surrounding bone in vivo could be advantageous to hydroxyapatite, which remains unremodeled despite long periods upon implantation (Ogose et al., 2005).

The design of these 3D fibrous scaffolds was expected to enhance the synergistic effects of ceramic (osteoconductivity and bioactivity) and biopolymer features (elasticity and shape control). The possibility to combine and stack layers in such simple and thin structures could improve their tridimensionality, which has been described to be critical in the structure, function, and morphology of body tissues.

The limited diffusion in standard static culture environments is another important issue to consider when designing bone regeneration strategies, since it could constrain cell proliferation and uniform distribution in tissue engineered constructs (Hosseinkhani et al., 2006; $\mathrm{Yu}$ et al., 2004). As a result, the current study also proposed to evaluate the effect of dynamic culturing in the osteogenic differentiation process of goat bone marrow stromal cells (gBMSCs) cultured in the multilayer scaffolds, either in the presence of basic or osteogenic differentiation media.

\section{Materials and methods}

\subsection{Development of the nanofibrous multilayered composite scaffolds}

The ceramic material, beta-tricalcium phosphate ( $\beta$-TCP), was obtained from a solid state reaction between stoichiometric amounts of calcium phosphate dibasic anhydrous (Fluka) and calcium carbonate (Sigma-Aldrich Quimica, S.A. Sintra, Portugal) followed by a $24 \mathrm{~h}$ sinterization at $800^{\circ} \mathrm{C}$. The powders were hand-sieved with stainless steel sieves (mesh 225-106 $\mu \mathrm{m}$ ). A polymeric solution of $17 \%(\mathrm{w} / \mathrm{v})$ polycaprolactone (PCL) in a mixture of chloroform (Aldrich) and N,N-dymethylformamide (Aldrich) at a 7:3 ratio was processed by electrospining technique. This solution was electrospun at $9-10 \mathrm{kV}$ with a flow rate of $1.0 \mathrm{ml} / \mathrm{h}$ collecting a random fiber mesh $(20 \mathrm{~cm}$ away from the collector) on a flat aluminium foil. Composite scaffolds were then developed by assembling three stacked layers of electrospun PCL fibers and TCPs at a ratio of $0.5 \mathrm{~g}$ TCP per PCL mesh. $0.25 \mathrm{~g}$ of TCP powder was dispersed between each two consecutive PCL layers. The resulting PCL-TCP scaffolds as well as TCPs powder and PCL meshes alone were characterized by FTIR, XRD, and SEM as described below.

\subsection{Characterization of the composite scaffolds}

\subsubsection{Thin-film X-ray diffraction (TF-XRD)}

TF-XRD patterns were recorded on a Philips X'Pert MPD (Philips, The Netherlands) diffractometer using $\mathrm{CuK} \alpha$ 
radiation to analyze the surface composition of the specimens.

\subsubsection{Fourier transformed infrared spectroscopy with attenuated total reflectance (FTIR-ATR)}

FTIR-ATR was performed using IRPrestige-21 equipment (Shimadzu, Japan). Spectra were taken with a resolution of $2 \mathrm{~cm}^{-1}$ and averaged over 64 scans, covering the wave number range of $4400-400 \mathrm{~cm}^{-1}$.

\subsubsection{Scanning electron microscopy (SEM)}

SEM analysis was performed to characterize the morphology of the developed structures using Hitachi S-2600 N equipment (Hitachi Koki CO, LTD, Japan). Prior to any SEM observations, sample surfaces were gold sputtered.

\subsubsection{In vitro culture of marrow mesenchymal cells onto nanofibrous scaffolds}

To assess cellular behavior, scaffolds were cut into 5-mm diameter discs and then sterilized by means of two 30-min cycles of UV irradiation. PCL nanofiber meshes without TCPs were considered as controls for the experiment.

Goat bone marrow stromal cells (gBMSCs) were harvested from iliac crests of adult goats and expanded in basal medium composed of DMEM (Dulbecco's Modified Eagle Medium, Sigma) supplemented with 10\% FBS (Invitrogen; Life Technologies S.A 28108 (Alcobendas, Madrid) and 1\% antibiotic/antimycotic solution (A/A) $\left(\right.$ Gibco $^{\circledR}$; Sigma-Aldrich), and then seeded onto the PCLTCP composites at a concentration of $5.0 \times 10^{4}$ cells/mesh or scaffold. After the seeding, cell-scaffold constructs were maintained in non treated 48 multi-well plates $\left(\right.$ Costar $^{\circledR}$; Sigma-Aldrich) in basal medium for $24 \mathrm{~h}$ and then cultured under static or dynamic conditions in either basal or osteogenic differentiation medium during 7, 14, or 21 days. Dynamic conditions were provided by an orbital shaker (Digisystem Laboratory Instruments Inc., Taipei Hsien, Taiwan) under mild constant agitation ( $60 \mathrm{rpm})$, working permanently until the end of the experiment and kept in the same $\mathrm{CO}_{2}$ incubator where constructs under static conditions were also cultured.

Osteogenic medium was prepared with $\alpha$-MEM (Minimal Essential Medium Eagle alpha modification, Sigma-Aldrich) $10 \%$ FBS and 1\% A/A, and osteogenic supplements, namely $50 \mu \mathrm{g} / \mathrm{ml}$ ascorbic acid, $10^{-8} \mathrm{M}$ dexamethasone, and $10 \mathrm{mM} \beta$-glycerophosphate (All from Sigma-Aldrich). In all conditions, cell culture medium was changed twice a week.

\subsubsection{Cell proliferation and osteogenic differentiation in multi-stacked nanofibrous scaffolds}

Cell proliferation was assessed by a DNA quantification assay while the osteogenic differentiation was assessed by ALP quantification, Alizarin Red staining, and immunocytochemistry (ICC) for osteocalcin (OC) and type I collagen.

DNA assay. Proliferation potential of gBMSCs seeded onto the developed scaffolds was considered by double strand DNA quantification (dsDNA). For this purpose, a fluorimetric dsDNA quantification kit (PicoGreen, Molecular Probes, Invitrogen) was used. The fluorescence was read using a microplate ELISA reader (BioTek, Winooski, VT, USA) at an excitation of $485 / 20 \mathrm{~nm}$ and an emission of $528 / 20 \mathrm{~nm}$.

ALP assay. ALP activity was measured in this study as an osteogenic cell marker. A substrate solution consisting of $0.2 \%$ (wt/v) p-nytrophenyl phosphate (Sigma-Aldrich) in a substrate buffer with $1 \mathrm{M}$ diethanolamine $\mathrm{HCl}$ (Merck, Lisbon, Portugal) at pH 9.8 was added to each sample. The plate was incubated in the dark for $45 \mathrm{~min}$ at $37^{\circ} \mathrm{C}$ followed by the addition of a stop solution ( $2 \mathrm{M}$ $\mathrm{NaOH}$ (Panreac; Barcelona, Spain) plus $0.2 \mathrm{mM}$ EDTA (Sigma-Aldrich). Samples and standards (the latter prepared with $10 \mu \mathrm{mol} . \mathrm{ml}^{-1}$ p-nitrophenol (Sigma-Aldrich)) were analysed in triplicates. The absorbance was read using a microplate ELISA reader (BioTek) at $405 \mathrm{~nm}$.

Alizarin Red staining. Cell-scaffold constructs were removed from culture and fixed in $4 \%$ formalin (SigmaAldrich) solution overnight at $4{ }^{\circ} \mathrm{C}$. A $2 \%$ Alizarin Red solution (Sigma-Aldrich) was prepared ( $\mathrm{pH}$ adjusted to 4.1-4.3) to stain calcium ions that might be present resultant from mineral ECM produced by cells. Constructs were stained with alizarin red solution for about $2 \mathrm{~min}$ and washed with PBS until removal of excess of stain and let dry.

Immunocytochemistry. After overnight fixation with 4 $\%$ formalin, samples were kept in PBS until immunocytochemistry (ICC) analysis. ICC was performed according to the PK 7200 R.T.U. Vectastain ${ }^{\circledR}$ Universal Elite ${ }^{\circledR}$ ABC kit from VectorLabs (Burlingam, CA, USA) using R.T.U Normal Horse Serum to avoid unspecific reactions, a biotinylated secondary antibody (R.T.U. Biotinylated Universal Antibody), and a Peroxidase Substract Kit (Vector SK-4100). Primary antibodies Collagen I (MAB339) (Chemicon International, Phillipsburg, NJ, USA) and Osteocalcin (Ab13418) (Abcam, Hong Kong, China) were prepared using a 1/100 dilution.

\subsection{Statistical analysis}

Statistical analysis was carried out using One Way ANOVA test and Tukey's Multiple Comparison Test $(p<0.05)$. All results are presented as mean \pm standard deviation. In the present study, controls of the experiment were considered using PCL nanofiber meshes without TCPs.

\section{Results and discussion}

Goat model is a popular animal model for orthopaedics, whose stem cells can be applied to autologous approaches in bone TE. Previous studies have demonstrated the 
potential of gBMSCs to differentiate into an osteogenic lineage (Oliveira et al., 2006; Leonor et al., 2011; Rodrigues et al., 2011). Furthermore, the PCL nanofiber meshes used in this study as positive controls for determining the applicability and improvement of multi-layered PCL-TCP scaffolds in bone-related strategies, have shown to promote cellular adhesion, proliferation, and osteogenic differentiation of mesenchymal stem cells (Binulal et al., 2010).

Multilayered composite scaffolds were achieved through the dispersion of TCP onto electrospun PCL fibers. The calcium phosphate obtained from the chemical reaction presented a rhombohedrical structure, typical of $\beta$-TCP, and the vibrational bands associated to the phosphate groups, as demonstrated by FTIR (Figure 1) and XRD (Figure 2) data.

SEM observation indicated a dispersion of TCPs granules within the electrospun PCL nanofibers structure in the produced composite scaffolds (Figure 3). A random distribution was observed not only with TCPs granules but also in nanofibers of the mesh-like stratified structure. Additionally, TCP mineral particles were integrated within the structure of multi-layered nanofiber scaffolds.

In this study, gBMSCs attached and proliferated into the PCL-TCP scaffolds both in static and dynamic conditions, as well as in basal and osteogenic culture conditions. Similar results were achieved in control PCL meshes. In terms of proliferation, gBMSCs showed two different behaviours $(p>0.05)$; cell proliferation in PCL meshes increased with time in culture, while in PCL-TCP constructs cells increased up to 14 days in basal or osteogenic culture conditions and then, the cell number decreased at week 3 (Figure 4).

This decreasing tendency of proliferation in PCL-TCP scaffolds could be a result of the osteogenic differentiation process undertaken by the gBMSCs. Some studies indicate an inverse relationship between proliferation and differentiation of osteoprogenitor cells during bone formation (Lian and Stein, 1992).

ALP activity levels (Figure 5) were not detected for samples cultured for 7 or 14 days, probably due to low basal concentrations of ALP enzyme in the samples. The

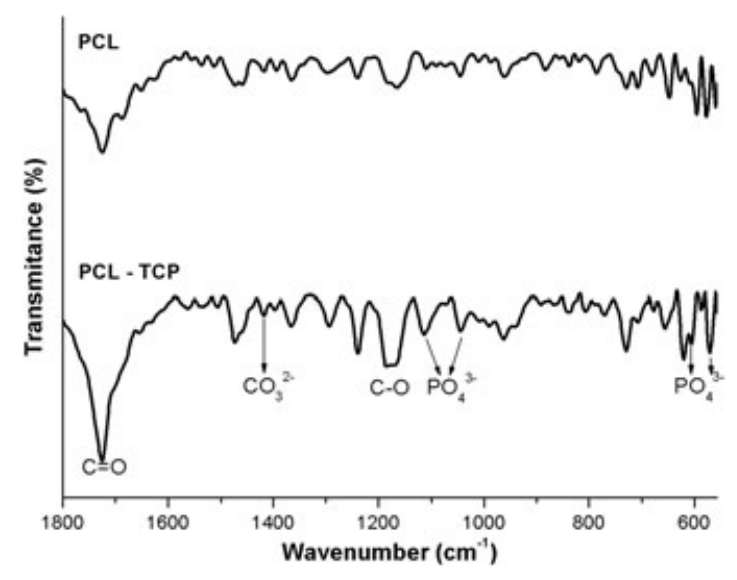

Figure 1. FTIR-ATR analysis of multilayered scaffolds. Membranes of PCL nanofiber mesh (PCL) were used as controls

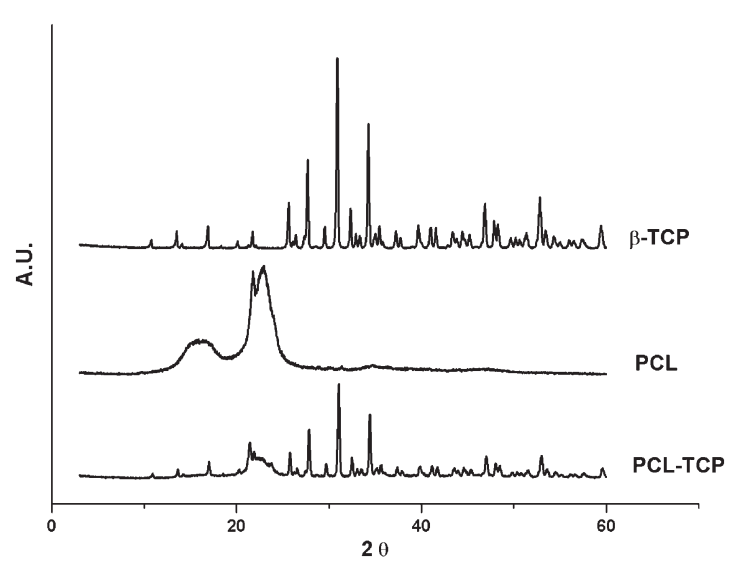

Figure 2. XRD analysis of multilayered scaffolds. Membranes of PCL nanofiber mesh (PCL) were used as controls

ALP/DNA ratio in PCL meshes cultured under dynamic conditions using osteogenic media was very similar to that found for PCL-TCP constructs cultured under static conditions in the same medium after 3 weeks in culture. Nevertheless, ALP was highly expressed in PCL-TCP constructs cultured under dynamic culture conditions and in osteogenic medium as compared to all the remaining study groups $(p<0.05)$. ALP activity data indicated a positive and synergistic effect of TCPs and mechanical stimulation on the development of ECM by osteogenically committed gBMSCs.

Furthermore, to confirm gBMSCs differentiation onto PCL-TCP scaffolds, osteogenic phenotype characterization was assessed by immunocytochemistry analysis for type I collagen and osteocalcin (OCa) and alizarin red staining for calcium detection after 21 days in culture (Figure 6).

Collagen I is the major protein present in bone ECM. All PCL-TCP scaffolds seeded with gBMSCs were positively stained for collagen I, although a more intense immunostain was observed in constructs cultured under dynamic conditions (Figure 6). In gBMSCs/PCL meshes (control), collagen I was more intense in basal medium culture. These results showed that a collagen I-rich ECM was being produced by gBMSCs either in PCL-TCP scaffolds or PCL meshes. In addition to a collagen matrix, a calcified milieu is an important part of the native bone tissue. Osteocalcin (OCa) is a non-collagenous protein produced by osteoblasts that participates in the matrix mineralization of bone (Lian et al., 1989b). OCa was found to be present in gBMSCs-PCL-TCP constructs under dynamic environment, while in static cultures OCa was only detected in the presence of osteogenic medium. Furthermore, in gBMSCs-PCL meshes (control), OCa was detected under static conditions in osteogenic differentiation medium (Figure 6).

Alizarin red staining was assessed to detect calcium ions, essential for the ECM mineralization. After 21 days in culture, gBMSCs-PCL-TCP constructs exhibited a stronger stain when compared to gBMSCs-PCL membrane controls (Figure 6). The stain was more intense under dynamic conditions both in basal or osteogenic media, as well as in constructs cultured in osteogenic differentiation 

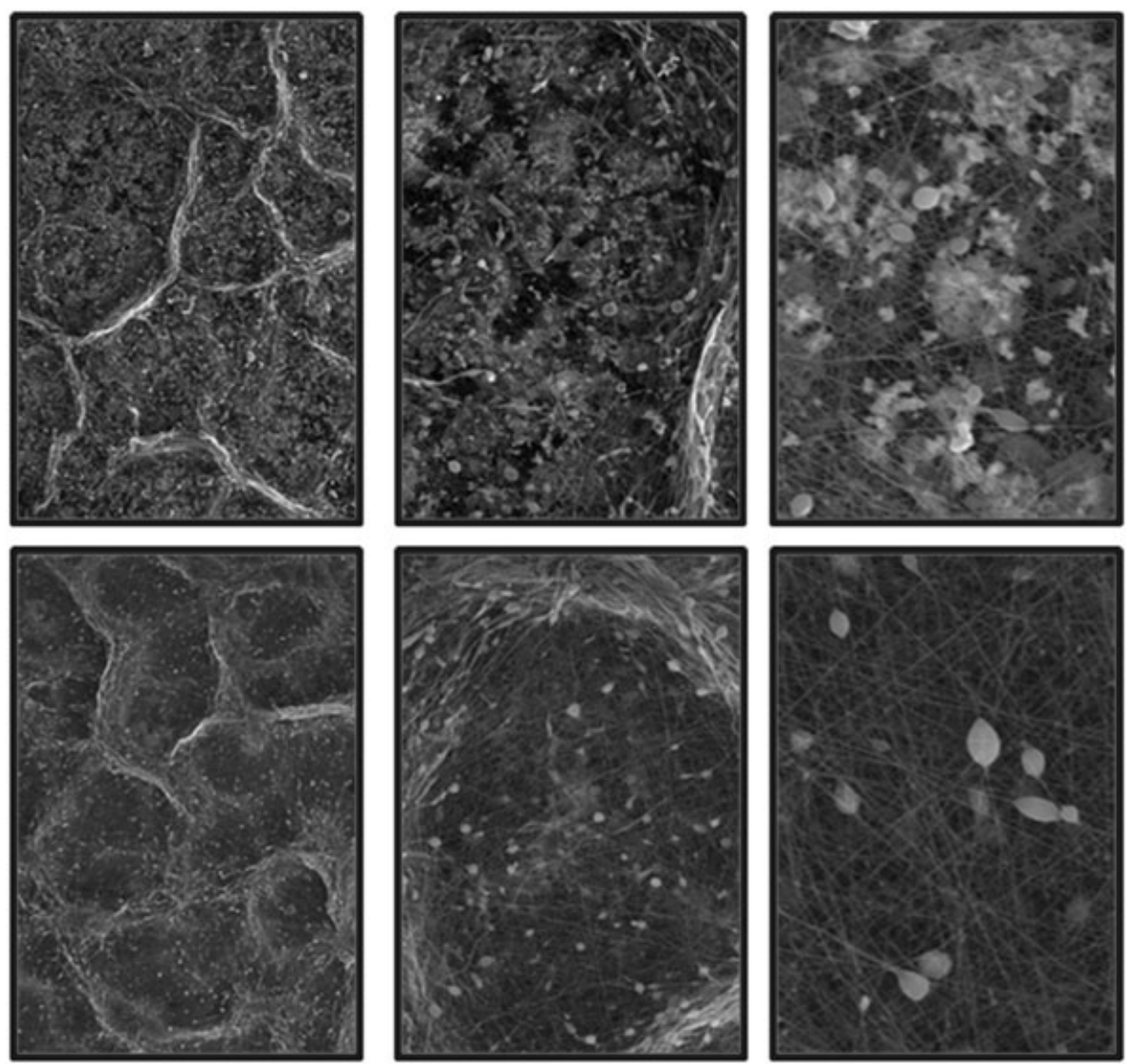

Figure 3. SEM analysis of multilayer scaffolds; the upper SEM micrographs refer to the PCL-TCP multilayer scaffolds while the lower pictures correspond to the control of PCL nanofiber mesh (left to right: $100 \mathrm{x}, 300 \mathrm{x}$, and $1000 \mathrm{x}$ magnifications)

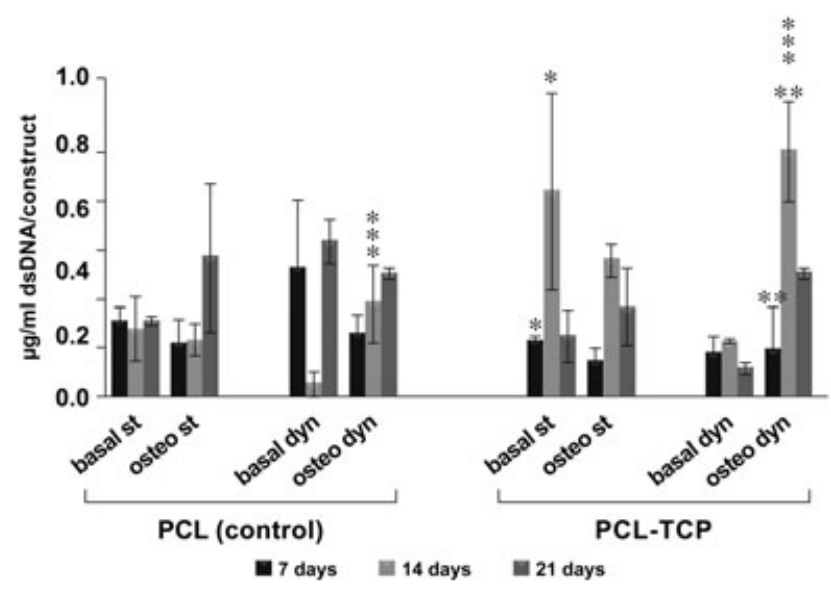

Figure 4. gBMSCs proliferation given by DNA quantification was assessed in PCL-TCP scaffolds and PCL membranes (control) after 7, 14, or 21 days in basal or osteogenic media in either static (st) or dynamic (dyn) conditions. Symbols *,**, and *** denote study groups with statistically significant differences $(p<0.05)$ as using One Way ANOVA method

medium in a static environment, indicating the production of calcified ECM. OCa and Alizarin Red staining followed a similar pattern in gBMSCs-PCL-TCP constructs, which confirms the formation of a mineralized ECM, as OCa is thought to bind to hydroxyapatite in a calcium-

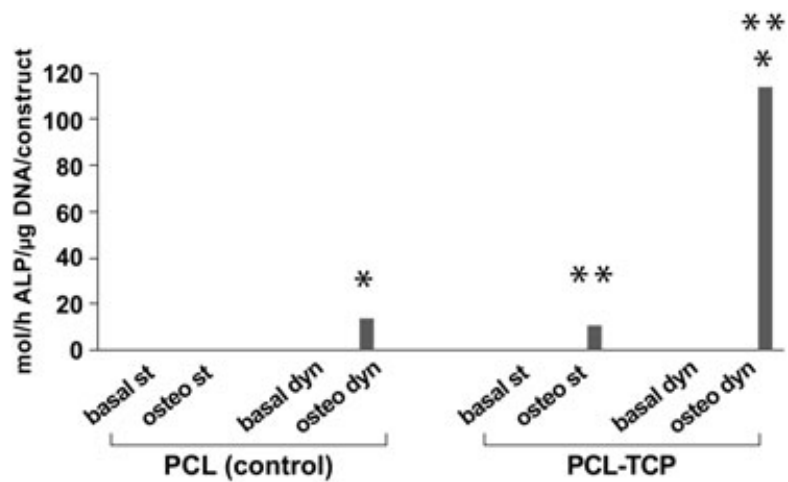

Figure 5. gBMSCs osteogenic differentiation given by ALP/DNA ratio was assessed in PCL-TCP scaffolds and PCL membranes (control) after 7, 14, or 21 days in basal or osteogenic media in either static (st) or dynamic (dyn) conditions. Symbols *, and ** denote groups with statistically significant differences $(p<0.05)$, as using One Way ANOVA method

dependent manner (Lian et al., 1989a) during the natural development of bone.

Even if in all culture conditions, gBMSCs-PCL-TCP constructs expressed osteogenic markers to some extent, the combination of these markers was stronger in dynamic conditions, especially for alizarin red staining. It is important to highlight that mineralization nodules (observed as darker red spots) are visible only in the 


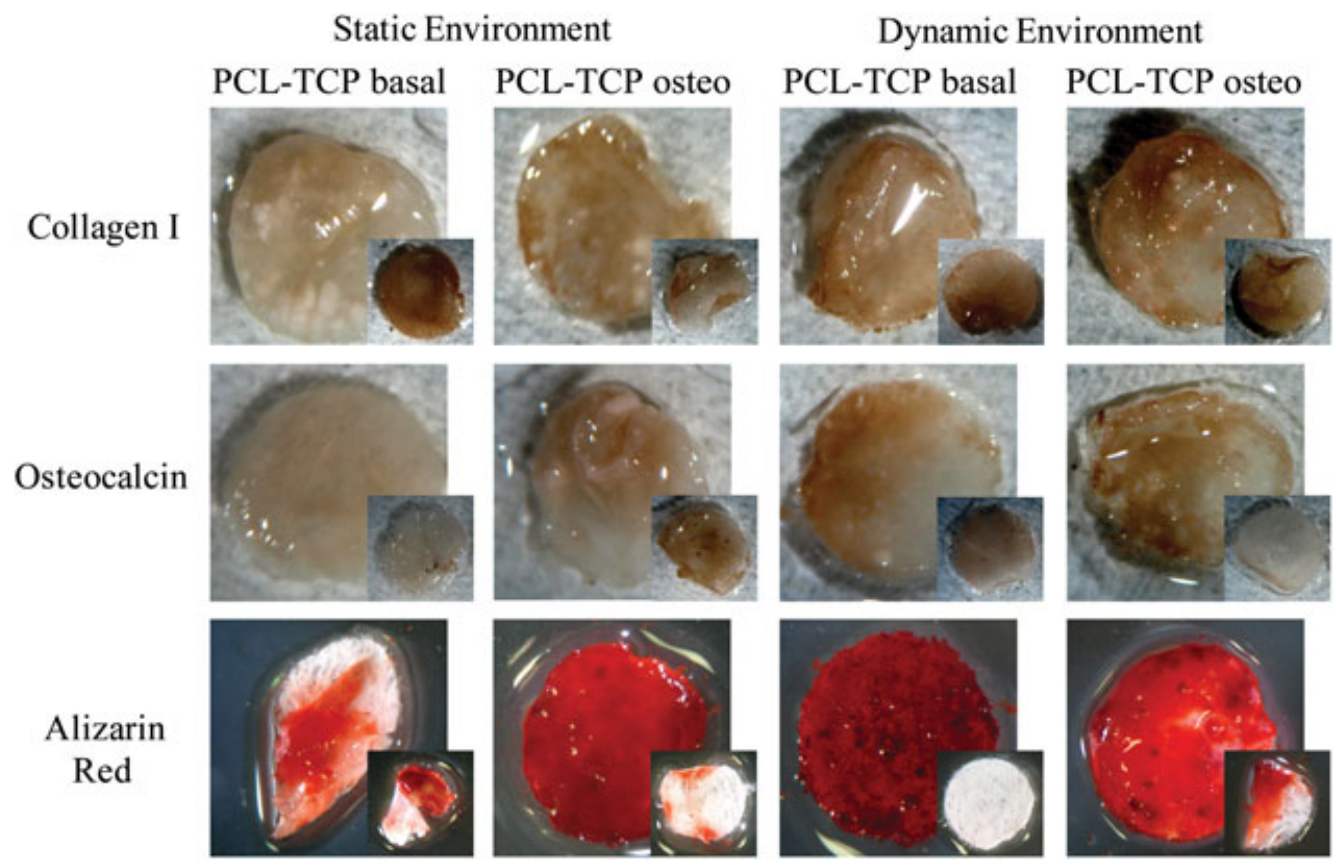

Figure 6. Osteogenic phenotype characterization of gBMSCs seeded onto PCL-TCP scaffolds after 21 days in culture with basal or osteogenic media either in static or dynamic conditions. Immunocytochemistry (ICC) was performed for collagen I and Osteocalcin (OCa) as well as Alizarin Red staining. Insets represent PCL meshes (control) under the same conditions

dynamic culture conditions and in the multilayer composite scaffolds, corroborating the previous results of ALP activity. The fact that ALP, an enzyme associated to ECM development and maturation (Lian and Stein 1992), was not expressed by cells seeded onto PCL-TCP constructs in basal/dynamic environment is quite interesting, since these cells are able to produce calcified ECM (Figure 6). Salasznyk et al. (2005) reported the bone isoform of ALP is found only in osteogenic supplemented mesenchymal stem cells, which justifies the basal ALP activity levels in basal/dynamic environment despite the mineralization of ECM. In their study, cells in basal medium did not differentiate when ALP was not expressed but the presence of PCL-TCP constructs could stimulate gBMSCs to select a different pathway in the absence of osteogenic medium that could be also encouraged by the dynamic environment, since calcified ECM is reduced in basal/ static conditions. Furthermore, in the presence of PCLTCP scaffolds, gBMSCs achieved osteogenic differentiation and ECM mineralization even in the absence of osteogenic supplements after 3 weeks in culture.

Mechanical stimuli are required to maintain a healthy bone and described to induce the up-regulation of osteogenic marker genes and increase matrix mineralization of the ECM in the presence of substrates coated with ECM proteins (Huang et al., 2009). Furthermore, cyclic mechanical stretching has also been described to participate in an increased ALP activity and mineralized matrix deposition (Huang et al., 2009). In the current study, the particular structure of PCL-TCP scaffolds mimicking bone natural ECM, both in structure and composition, stimulated gBMSCs to produce ECM matrix (collagen I and OCa). Under dynamic conditions, the environment induced by PCL-TCP scaffolds was sufficient to promote osteogenic differentiation even in basal medium conditions.

In summary, PCL-TCP scaffolds participate in the osteogenic process of gBMSCs even in basal medium and static conditions. Nevertheless, the osteogenic potential is enhanced in dynamic environments, evidencing the synergistic effect of TCPs and mild mechanical stimulation in gBMSCs osteogenic phenotype.

\section{Conclusions}

The production of multi-layered PCL-TCP scaffolds was successfully achieved as well as the in vitro assessment of gBMSC-PCL-TCP constructs aiming at bone tissue strategies. Moreover, considering that bone ECM is essentially an organic-inorganic composite and nano-scaled organized, the developed multilayered composite could be a promising system in the regenerative medicine field.

The results obtained suggest that the combination of PCL with calcium phosphates generated scaffolds with improved capacity to promote gBMSCs adhesion, proliferation, and expression of osteogenic phenotype, when compared to the synthetic polymer alone. Furthermore, the dynamic environment positively affected the gBMSCs behaviour and enhanced gBMSCs phenotypic expression and mineralized matrix synthesis. Interestingly, the synergistic effect of mild agitation and PCL-TCP scaffolds stimulated gBMSCs differentiation and production of a calcified ECM even in the absence of osteogenic supplements from the culture medium.

The development of PCL nanofibers with ceramic materials in a multilayered structure is an innovative methodology with great potential for the development 
of more adequate scaffolds for bone tissue engineering Acknowledgements approaches.

\section{Conflict of interest}

Authors declare no conflict of interest.
M. T. Rodrigues thanks the Portuguese Foundation for Science and Technology (FCT) for providing a PhD scholarship (SFRH/BD/30745/2006). This work was carried out under the scope of the European NoE EXPERTISSUES (NMP3-CT2004-500283).

\section{References}

Araujo JV, Martins A, Leonor IB et al. 2008; Surface controlled biomimetic coating of polycaprolactone nanofiber meshes to be used as bone extracellular matrix analogues. J Biomater Sci Polym Ed 19(10): 1261-1278.

Bezwada RS, Jamiolkowski DD, Lee IY et al. 1995; Monocryl(R) Suture, a New UltraPliable Absorbable Monofilament Suture. Biomaterials 16(15): 1141-1148.

Binulal NS, Deepthy M, Selvamurugan N et al. 2010; Role of Nanofibrous Poly (Caprolactone) Scaffolds in Human Mesenchymal Stem Cell Attachment and Spreading for In Vitro Bone Tissue Engineering-Response to Osteogenic Regulators. Tissue Eng Part A 16(2): 393-404.

Chen GP, Ushida T, Tateishi T. 2002; Scaffold design for tissue engineering. Macromol Biosci 2(2): 67-77.

Collins AM, Skaer NJV, Gheysens T et al. 2009; Bone-like resorbable silkbased scaffolds for load-bearing osteoregenerative applications. Adv Mater 21 75-78.

Gupta D, Venugopal J, Mitra S et al. 2009; Nanostructured biocomposite substrates by electrospinning and electrospraying for the mineralization of osteoblasts. Biomaterials 30(11): 2085-2094.

Hosseinkhani H, Hosseinkhani M, Tian F et al. 2006; Ectopic bone formation in collagen sponge self-assembled peptideamphiphile nanofibers hybrid scaffold in a perfusion culture bioreactor. Biomaterials 27(29): 5089-5098.

Huang CH, Chen MH, Young TH et al. 2009; Interactive effects of mechanical stretching and extracellular matrix proteins on initiating osteogenic differentiation of human mesenchymal stem cells. $J$ Cell Biochem 108(6): 1263-1273.

Ko EK, Jeong SI, Rim NG et al. 2008; In vitro osteogenic differentiation of human mesenchymal stem cells and in vivo bone formation in composite nanofiber meshes. Tissue Eng Part A 14(12): 2105-2119.

Leonor I, Rodrigues MT, Gomes ME et al. 2011; In Situ Functionalization of WetSpun Fibre meshes for Bone Tissue Engineering: One Step Approach. J Tissue Eng Regen Med 5: 104-111.
Li C, Vepari C, Jin HJ et al. 2006; Electrospun silk-BMP-2 scaffolds for bone tissue engineering. Biomaterials 27(16): 3115-3124.

Li M, Mondrinos MJ, Gandhi MR et al. 2005a; Electrospun protein fibers as matrices for tissue engineering. Biomaterials 26(30): 5999-6008.

Li WJ, Tuli R, Huang X et al. 2005b; Multilineage differentiation of human mesenchymal stem cells in a three-dimensional nanofibrous scaffold. Biomaterials 26(25): 5158-5166.

Lian J, Stewart C, Puchacz E et al. 1989a; Structure of the Rat Osteocalcin Gene and Regulation of Vitamin-D-Dependent Expression. Proc Natl Acad Sci USA 86(4): 1143-1147.

Lian JB, Stein GS, Stewart C et al. 1989b; Osteocalcin - Characterization and Regulated Expression of the Rat Gene. Connect Tissue Res 21(1-4): 391-399.

Lian JB, Stein GS. 1992; Concepts of osteoblast growth and differentiation: basis for modulation of bone cell development and tissue formation. Crit Rev Oral Biol Med 3(3): 269-305.

Martins A, Reis RL, Neves NM. 2008; Electrospinning: processing technique for tissue engineering scaffolding. Int Mater Rev 53(5): 257-274.

Ngiam M, Liao S, Patil AJ et al. 2009; Fabrication of mineralized polymeric nanofibrous composites for bone graft materials. Tissue Eng Part A 15(3): 535-546.

Ogose A, Hotta T, Kawashima $\mathrm{H}$ et al. 2005; Comparison of hydroxyapatite and beta tricalcium phosphate as bone substitutes after excision of bone tumors. $J$ Biomed Mater Res B Appl Biomater 72(1): 94-101.

Oliveira JM, Rodrigues MT, Silva SS et al. 2006; Novel hydroxyapatite/chitosan bilayered scaffold for osteochondral tissueengineering applications: Scaffold design and its performance when seeded with goat bone marrow stromal cells. Biomaterials 27(36): 6123-6137.

Rezwan K, Chen QZ, Blaker JJ et al. 2006; Biodegradable and bioactive porous polymer/inorganic composite scaffolds for bone tissue engineering. Biomaterials 27(18): 3413-3431.
Rodrigues MT, Gomes ME, Viegas CA et al. 2011; Tissue Engineered Constructs based on SPCL Scaffolds Cultured with Goat Marrow Cells: Functionality in Femoral Defects. J Tissue Eng Regen Med 5(1): 41-49.

Salasznyk RM, Klees RF, Westcott AM et al. 2005; Focusing of gene expression as the basis of stem cell differentiation. Stem Cells Dev 14(6): 608-620.

Sefcik LS, Neal RA, Kaszuba SN et al. 2008; Collagen nanofibres are a biomimetic substrate for the serum-free osteogenic differentiation of human adipose stem cells. $J$ Tissue Eng Regen Med 2(4): 210-220.

Shin M, Yoshimoto H, Vacanti JP. 2004; In vivo bone tissue engineering using mesenchymal stem cells on a novel electrospun nanofibrous scaffold. Tissue Eng 10(1-2): 33-41.

Srouji S, Kizhner T, Suss-Tobi E et al. 2008; 3-D Nanofibrous electrospun multilayered construct is an alternative ECM mimicking scaffold. J Mater Sci Mater Med 19(3): 1249-1255.

Tsuji H, Ikada Y. 1996; Blends of aliphatic polyesters. 1. Physical properties and morphologies of solution-cast blends from poly(DL-lactide) and poly(epsiloncaprolactone). J Appl Polym Sci 60(13): 2367-2375.

Xin X, Hussain M, Mao JJ. 2007; Continuing differentiation of human mesenchymal stem cells and induced chondrogenic and osteogenic lineages in electrospun PLGA nanofiber scaffold. Biomaterials 28(2): 316-325.

Yang S, Leong KF, Du Z et al. 2001; The design of scaffolds for use in tissue engineering. Part I. Traditional factors. Tissue Eng 7(6): 679-689.

Yu X, Botchwey EA, Levine EM et al. 2004. Bioreactor-based bone tissue engineering: the influence of dynamic flow on osteoblast phenotypic expression and matrix mineralization. Proc Natl Acad Sci USA 101(31): 11203-11208.

Zhang YZ, Su B, Venugopal J et al. 2007; Biomimetic and bioactive nanofibrous scaffolds from electrospun composite nanofibers. Int $J$ Nanomedicine 2(4): 623-638. 